
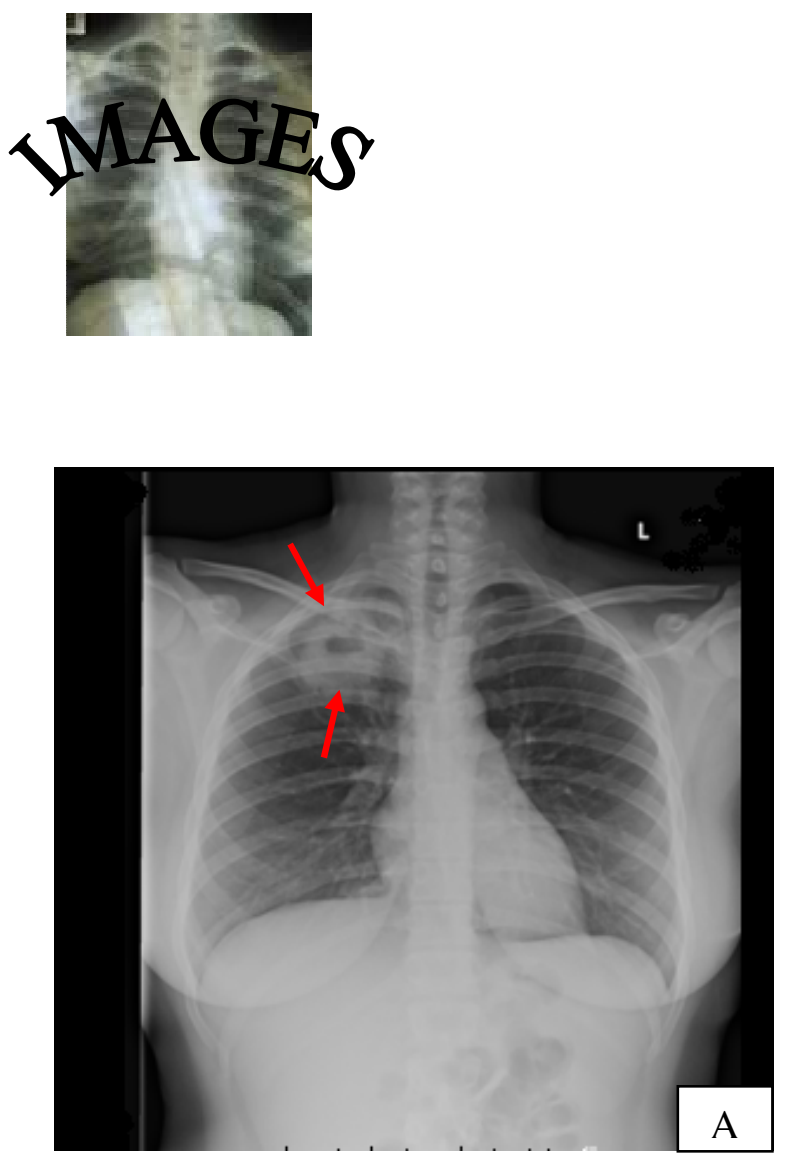

\section{Lung Abscess}

Furqan S. Siddiqi, M.D., ${ }^{1}$

Stephen Hagan, M.D. ${ }^{1,2}$

${ }^{1}$ University of Kansas

School of Medicine-Wichita

Department of Internal Medicine

${ }^{2}$ Private Practice, Wichita, KS

A 23-year-old female presented with a two-week history of worsening shortness of air, rightsided chest heaviness, and cough. The cough was initially dry, but later productive with foul smelling yellowish sputum. The patient denied fever, history of tuberculosis contact, or recent travel. Her physical examination showed stable vital signs and right upper lobe crackles. A complete blood count and comprehensive metabolic panel were normal. Blood and sputum cultures and a QuantiFERON ${ }^{\circledR}$-TB Gold for tuberculosis were negative. There were no HIV antibodies. Chest radiograph showed a 6-cm cavitary lesion in right upper lobe (see arrows in Figure A). A computer tomography (CT) of the chest showed $4.9 \times 5.4 \mathrm{~cm}$ necrotic lesion with thin and smooth walls in right upper lobe (see arrows in Figure B). A diagnosis of lung abscess was made and IV clindamycin was started. Acid-fast bacillus smear, fungal, bacterial cultures, and cytology on bronchoalveolar lavage and bronchial brushing were negative. The patient responded well to antibiotic treatment and later was switched to oral clindamycin. She was discharged receiving a total of six weeks of antibiotics.

\title{
Discussion
}

Lung abscess is defined as a necrotic lesion in lung parenchyma that contains pus. ${ }^{1}$ Although the necrotizing infections are the most important cause, other important pathogenetic factors include cavitations of the necrotic center of malignant tissue or necrosis of infracted lung tissue. Differentiating lung abscess from empyema on imaging can be challenging. In empyema, the CT of the chest typically shows thickened parietal and visceral pleura separated by fluid, known as the "split pleura sign" (Figure C). ${ }^{2}$ Fluid usually is organized in oblong fashion which 
displaces surrounding lung tissue away from fluid collection. The usual CT findings of a lung abscess include a spherical cavity with a thick wall that destroys the surrounding lung with bronchograms traveling into the abscess rather than displaced like in empyema. ${ }^{2-6}$ Color Doppler ultrasound also has shown to be a powerful tool to differentiate air fluid level in lung abscess from empyema. ${ }^{7}$

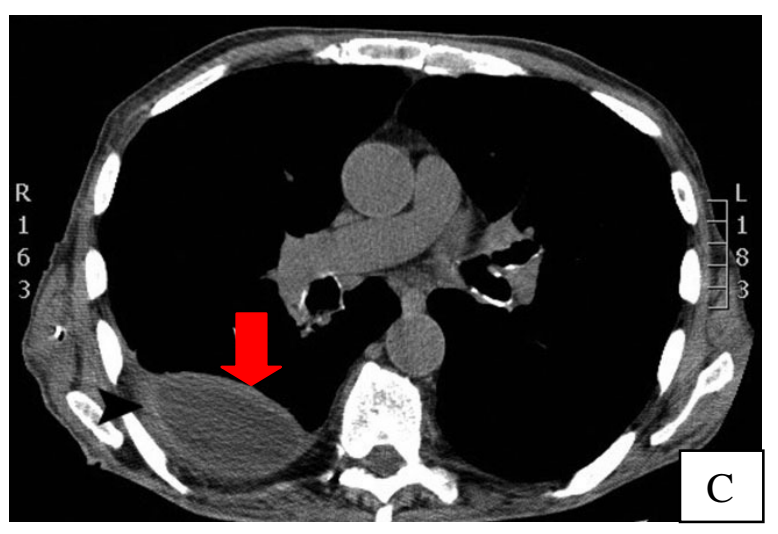

A CT image demonstrating thickening and enhancement of the parietal pleura and visceral pleura with fluid in between (i.e., the “split pleura sign'”).

\section{References}

${ }^{1}$ No authors listed. Lung abscess - Medical Staff Conference, University of California, San Francisco. West J Med 1976; 124(6):476-482. PMID: 936601.

2 Reynolds JH, McDonald G, Alton H, Gordon SB. Pneumonia in the immunocompetent patient. Br J Radiol 2010; 83(996):998-1009. PMID: 21088086.

3 McLoud TC, Flower CD. Imaging the pleura: Sonography, CT, and MR imaging. AJR Am J Roentgenol 1991; 156(6):1145-1153. PMID: 2028857.

${ }^{4}$ Müller NL. Imaging of the pleura. Radiology 1993; 186(2):297-309. PMID: 8421723.

${ }^{5}$ Godwin JD. Computed tomography of the chest. Philadelphia, Pa: Lippincott Williams and Wilkins, 1984; pp. 130-137. ISBN: 0397505914.

6 Reed A, Shogan P, Folio L. Radiology corner: Thoracic empyema versus lung abscess. Mil Med 2010; 175(9): 701-702. PMID: 20882937.

7 Chen HJ, Yu YH, Tu CY, et al. Ultrasound in peripheral pulmonary air-fluid lesions. Color Doppler imaging as an aid in differentiating empyema and abscess. Chest 2009; 135(6):14261432. PMID: 19255298.

Key Words: lung abscess, empyema 